\title{
Analysis of the Real-time Compensation for Thermal Error at CNC Milling Machine
}

\author{
Tsung-Chia Chen ${ }^{\text {a }}$ and Chia-Jung Chang \\ National Chin-Yi University of Technology, Dept. of Mechanical Engineering, 41170 Taichung, Taiwan (R.O.C.)
}

\begin{abstract}
This paper focuses on analyzing and discussing thermal errors at $\mathrm{CNC}$ milling machines. The thermal affection makes the deformation of machine tools and is the main problem of accuracy error over than $65 \%$. Effectively improving or controlling thermal errors is helpful for the accuracy of machine. The key point of this paper is the position of tool center point. Firstly, 14 pieces of temperature sensors are used for checking the real field of temperature, and then four sensors with better linearity are chosen for real situations. The test bar and 5 pieces of noncontact sensors are utilized for clearly getting the displacement of the tool center point and head during the process. Based on the theory of MRA (Multiple Regression Analysis), the external zero-point is shifted to build the mathematical module. The database is input to the control board and the PLC is used for real-time compensation for machining. Finally, two work pieces (one compensated and one non-compensated) are tested and compensated one presents better precision.
\end{abstract}

\section{Introduction}

Taiwan is a famous country for manufacturing CNC machines in the world, especially is good at the quality and price. Regarding the rising sales in China, South Korean, and countries in south-east Asia during the recently years, the marketing of Taiwan is decreasing. By the way, like the advanced countries, Swiss, Japan, Germany has been developed machining of intelligent, compensation in-time that is bases on high quality and efficacy for many years. It means that there is more and more competitiveness in the world and Taiwan must follow such a trend. That's why the academia and manufacturers need to work with each other to reach the goal. Although the 3 -axis center is the basic one, there is more space to improve and advance. In local famous companies, the technology could reach the geometry accuracy less than $0.015 \mathrm{~mm}$ (based on ISO 230 rule). Regarding the mechanism of machine, it is built by complicated parts, transmission, and electronic elements. Utilizing tradition theories to model, control and analyze the error of kinematic isn't easily.

The heat is the biggest factor in making errors because of the heat deformation and displacement which will directly cause bad quality of work-piece. For this reason, high accuracy of $\mathrm{CNC}$ machines is only the basic standard. How to reach high reliability and stability for precision work-piece is the customer need.

Recently years for the static, dynamic, and the error of the heat on machine tools, that as following to reference. Mr. Ramesh, Mannan \& Poo [1] indicated that the most important was to consider the accuracy of machine and focus on the error of geometry accuracy, heat, and shear force to explain lots of errors in detail. Finally, for utilizing sensors to gather great data for error compensation, the cheaper and easier way under the value of the precision and allowance is expected. The authors [2] discussed the error of heat and pointed out that when a machine worked continuously, the heat of moving parts and mechanism unit would cause errors. Using FEA and HTM models, there are three steps to model, measure and compensate for general compensation of heat in time, depending on PC system to control the compensation of machine directly. Although the technology needs improvement, especially in heat errors, it will become the standard equipment of advance machines in the future. To overcome limits to micron machines, Mr. Ha, Liu \& Yang [3] used a capacitance sensor, which was developed to measure the geometry accuracy, to measure every axis and vertical straightness led to the coordination. Multiple degrees of freedom measurement have been realized to measure 5 components of geometry of work-piece errors at the same time. Totally 18 errors of geometry have been successfully measured by utilizing least squares method. Result in the error of horizontal decreases $1.2 \mathrm{~mm}$ and the error of straightness to $0 . \mathrm{Mr}$. Wu \& Gong [4] used the thermocouple and non-contact displacement gauge to measure the temperature and thermal deformation in a gantry machine. Multiple Regression Analysis is utilized for building the mathematical model of temperature difference and thermal deformation. After the compensation simulation, the error of spindle that could be controlled in a certain range is acquired to highly prove the upgraded precision of work-piece. This method

\footnotetext{
a Corresponding author: ctchen@ncut.edu.tw
} 
is predictive and accurate reliably. Mr. Lee and Yang [5] studied machine tools with the optimal statistics and evaluation, it be analysis by continuous and least squares regression. That could be applied to the actual variable selection effectively, and several related groups could decrease the variable problems to improve the multiple regression, so it is effectively shorter calculates and is more reliable of model. To develop a system of thermal compensation in time for the application of thermal model, Mr. Vanherck, Dehaes and Nuttin [6] used the artificial neural network model to access the relation of multiple regressions and effectively compensate the thermal deformation of multiple axes' machine tools. The back-propagation calculation is used for deducting the maximum deformation from $0.15 \mathrm{~mm}$ to $0.015 \mathrm{~mm}$ and finally machining two work-pieces. One is compensated, and the other is non-compensated. This way could shorten the error from $0.075 \mathrm{~mm}$ to $0.016 \mathrm{~mm}$ and the time for trying error by vanishing to decrease the thermal error. For the tool center point, Mr. Horejša, Mareša, and Novotnýa [7] used MLR and TTF to develop an advanced thermal compensation in horizontal 4-axis machine tools. Different from original 21 sensors, 4 are utilized by multiple regressions. This model is different from general TTF, which was built up based on the operation of spindle. It effectively decreases the error from $0.10 \mathrm{~mm}$ to $0.01 \mathrm{~mm}$ and shortens the displacement of TCP, better than the original $75 \%$.

\section{Theory and application of heat}

\subsection{Heat transmission}

Generally, there are three ways to transmit heat.

1. Heat Conduction: Solid, heat conducts from higher temperature to lower.

$$
q=-k a \frac{d X}{d T}
$$

$q$ is heat conduction, $k$ is factor, $a$ is the area of interface, $d X$ is the distance, $d T$ is temperature of each side.

2. Heat convection: The fluid or air, it delivers naturally to affect volume and density by itself.

$$
q=h A \Delta T
$$

$q$ is heat convection, $h$ is factor, $A$ is sectional area, $\Delta T$ is temperature difference.

3. Heat irradiation: It delivers via electromagnetic wave and doesn't depend on anything.

$$
q=\sigma A\left(T_{1}^{4}-T_{2}^{4}\right)
$$

$\sigma$ : Stefan-Boltzmann constant $\left(5.669 \times 10^{-8} \mathrm{w} /\left(\mathrm{m}^{2} \mathrm{k}^{4}\right), A\right.$ : sectional area, $T_{1}$ and $T_{2}$ is the temperature of difference for two irradiation.

\subsection{Heat source of machine tools}

There are three kinds of heat source for the heat deformation (Figure $1 \&$ Figure 2). Firstly, the heat deformation of machine tools (roughly $65 \sim 70 \%$ ), include spindle rotation, ball-screw expanded, heat displacement, and heat bend of machine tools. Secondly, the error of volume of machine tools (roughly 30\%), include static bend, error of building, and error of mechanism. Finally, error of controlled system of machine tools (roughly 5\%), it's from the error of interpolation of $\mathrm{CNC}$ controllers or the error of servo systems. Anyway, the above needs to be overcome for recent precision manufacturing industry.
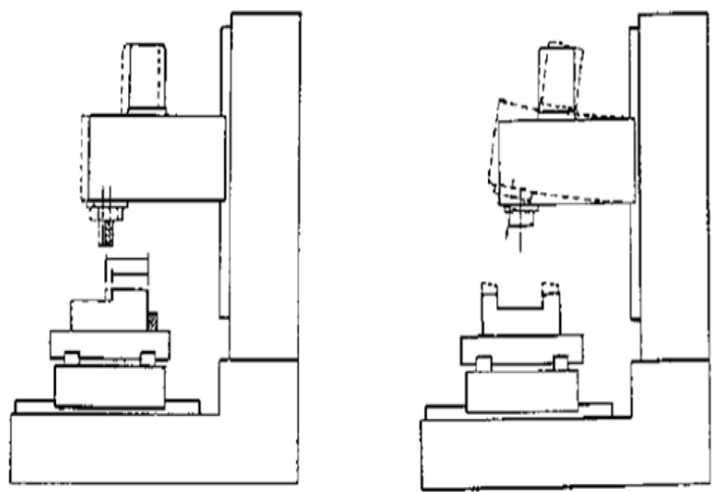

Figure 1. Heat deformation of machine tools.

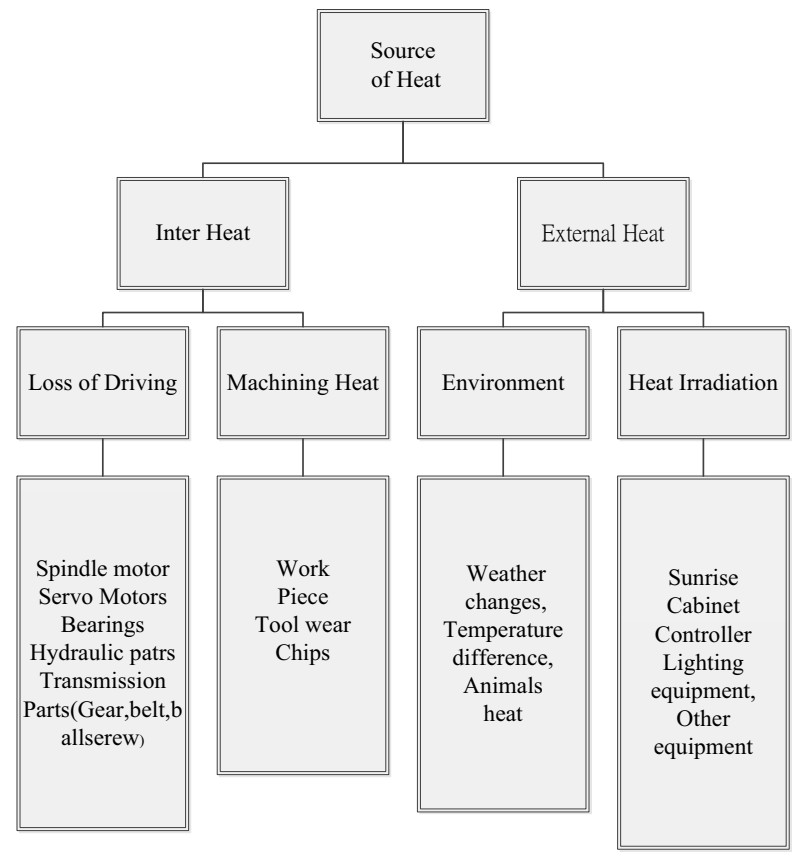

Figure 2. Factors in thermal deformation.

\subsection{Model building of thermal error}

There are two ways for building models.

\subsubsection{Theory model}

By analyzing the heat transfer, differential formula are utilized for getting the temperature of machine and then 
finding deformation based on the theory of elasticity, including data and numerical answers. It needs to transfer the numbers to show the mechanism and the heat source of machine. It is hard to explain the heat situation of machines accurately, because the mechanism is very complicated.

\subsubsection{Actual model}

It's an actual method to think that the machine system is a black box and to input the data of temperature about machine and other variables. Outputs are the deformation of machine tools or main factor of thermal errors in the system. It uses the parameters of system for the operation, like statistics model, exponential function, multiple regression, non-linear multiple regression, neural networks, expert system and fuzzy theory.

\section{Experiment}

\subsection{Experiment plan}

This paper uses 14 pieces of temperature sensors to measure the real field of temperature of the machine (Figure 3). Firstly, stick them on the machine to check the request. After the confirmation, fix 4 better relation sensors in the machine for gathering correct data as the following picture (Figure 4). Then, 4 pieces of noncontact displace sensors are used for checking the changeability of $\mathrm{X}, \mathrm{Y}, \mathrm{Z}$ axis.

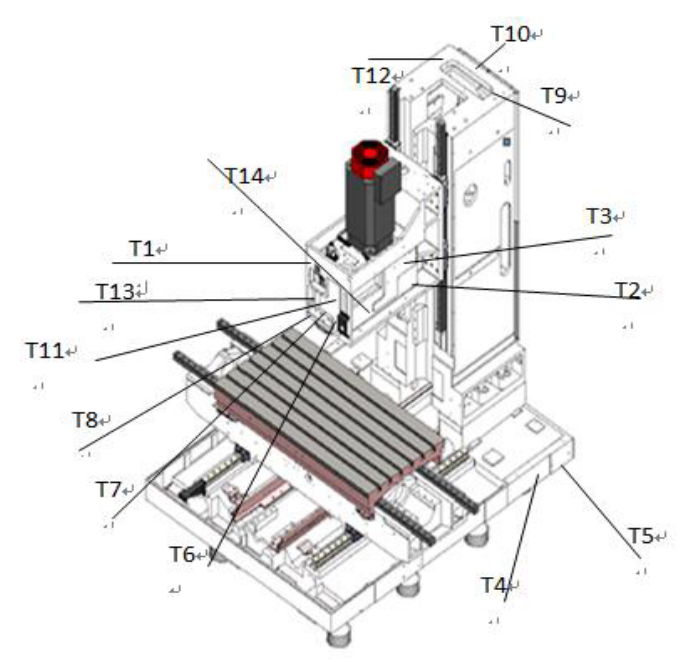

Figure 3. Position of sensors first.

After measuring the spindle running (7200RPM \& 10800RPM) for $48 \mathrm{hrs,} \mathrm{it} \mathrm{actually} \mathrm{needs} 4$ pieces of sensors to fix in good relations (Table 1) and then start to gather data.

Table 1. Point sensors.

\begin{tabular}{|l|l|}
\hline$T_{1}$ & Nearby rear side bearing of spindle \\
\hline$T_{2}$ & Heat source of belt transmission in head \\
\hline$T_{3}$ & Nearby spindle motor (directly) \\
\hline$T_{4}$ & Base, temperature of environment \\
\hline
\end{tabular}

\subsection{Equipment}

The Qauser machine tools, MV204C type (Figure 5), with the specification of Heidenhain Itnc530 controller, 12000RPM spindle (belt type), THK linear way (X, Y baller, $\mathrm{Z}$ roller), and auto-lubrication system, is used

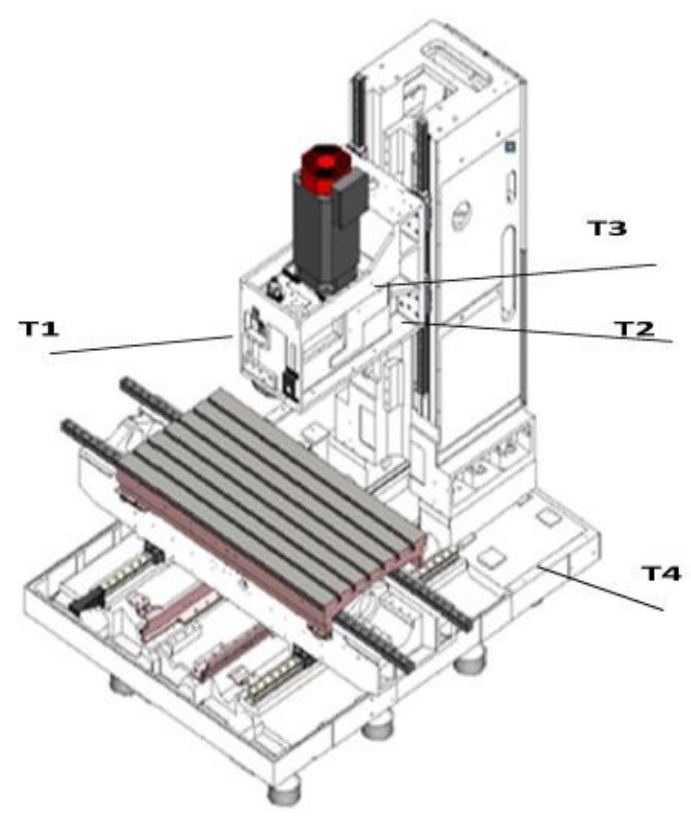

Figure 4. Position of needed sensors.

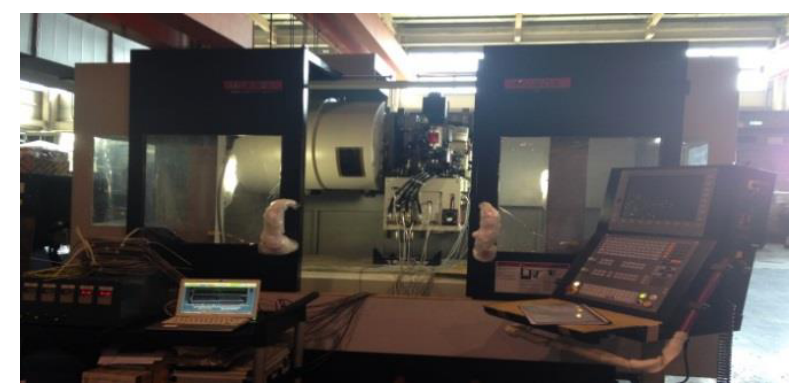

Figure 5. MV204C Quaser machine tools.

To compensate the microprocessor chip module (Figure 6), deliver through the PCB817 optical coupling electricity to PLC interface. (IO)

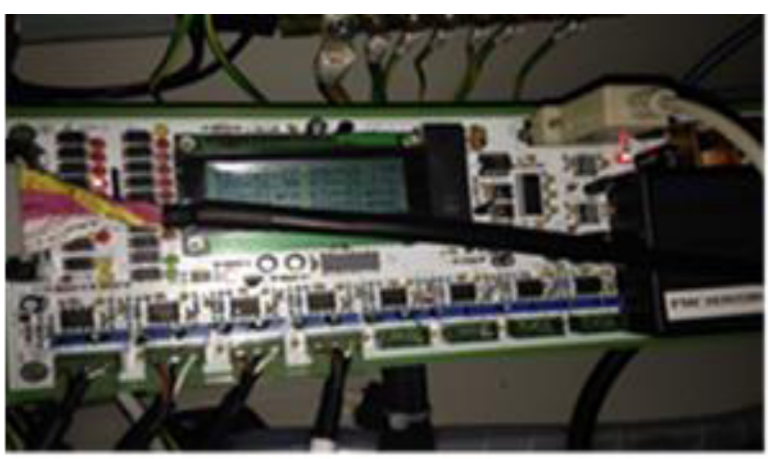

Figure 6. Compensation module. 


\section{Result of analysis and discuss}

\subsection{Displacement of temperature measuring}

Firstly, use 14 sensors to fit on the machine. Spindle runs 7200 RPM $(60 \%)$ and then the higher speed to 10800 RPM (90\%). After 48hrs, the data show steady state. Then, choose 3 good related sensors, like above ways, to operate again and get the data as following (Figure 7).

\subsection{Temperature changes in the amount of displacement}

From Figure 7, the displacement of $\mathrm{X}$ and $\mathrm{Y}$ axis is firstly run less than $0.02 \mathrm{~mm}$, and the position to the tool center is extended (TCP drift). Factors should be the bearing of oil being solid and running unstable, friction of bearing, or not being built well. After running, the chart shows in steady state within $0.055 \mathrm{~mm}, 90 \%$ RPM operation, the tool center point extends to $0.073 \mathrm{~mm}$ and then becomes steady. The displacement of $\mathrm{X}$ axis is roughly $0.01 \mathrm{~mm}$, meaning that the head of $\mathrm{X}$ axis tilts right side $0.01 \mathrm{~mm}$ (X1, X2) by thermal effect. The displacement of $\mathrm{Y}$ axis is roughly $0.016 \mathrm{~mm}(\mathrm{Y} 1, \mathrm{Y} 2)$, meaning the head is the rear side of 0.016 by error of accuracy. T1 is near the rear side of bearing and it's the biggest heat source; T3 is the operation of spindle motor; $\mathrm{T} 2$ is the friction of belt between pulleys; and, T4 is the temperature of environment. (Air condition factory, temperature is controlled in $28^{\circ} \mathrm{C}$ )

\subsection{Machining test}

After several times of compensation, the displacement of $\mathrm{X}, \mathrm{Y}$ axis is less than $0.02 \mathrm{~mm}$, and the point of grinding test bar is also within $0.02 \mathrm{~mm}$. Starting test, one is compensated and the other one is non-compensated.

Machining parameters contain $120 \mathrm{~mm}$ tool length, R3 ball tool, material being aluminum, and size of workpiece $200 \mathrm{~mm}$ X $200 \mathrm{~mm}$ X 50mm. Radial machine, from center to outside, consumes time for more than two hours.

Off the compensation, the face of accuracy is $0.025 \mathrm{~mm}$ (min.) and $0.021 \mathrm{~mm}$ (max.). After the compensation, the accuracy is 0.014 (min.) and $0.001 \mathrm{~mm}$ (max.). The effect rises $33 \%$ that the accuracy is absolutely more precise (as shown Figure. 8).

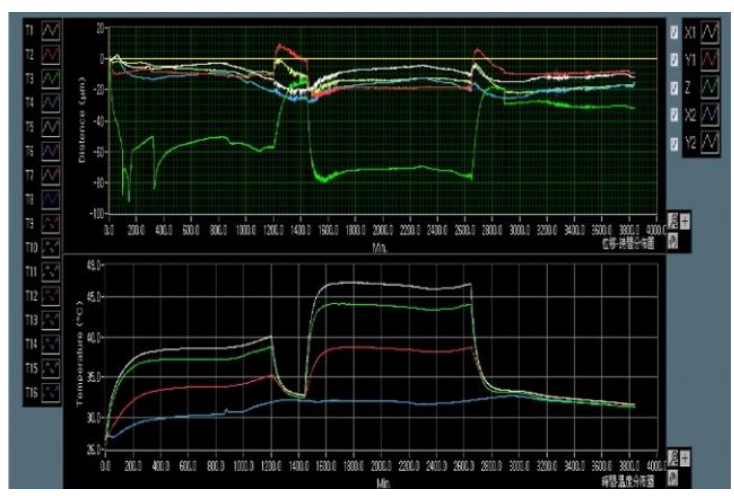

Figure 7. Temperature and displacement.
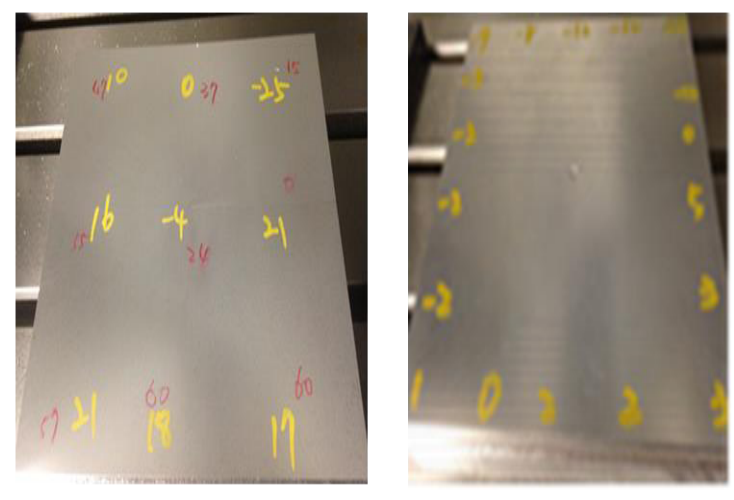

Figure 8. (a) Non-compensation. (b) Compensation.

\section{Conclusions}

After the experiment, the effects of heat on the error of accuracy for machine are very big, especially in deformation and displacement of tool point. The result of work-piece is apparent that it is concluded as below.

1. Mechanism of machine includes five parts of casting, where good heat balance and symmetrical design are very important. They could lower the error of heat, when they are well designed.

2. It's very important for the position of sensors. The experiment shows that too many sensors will lead to complex and unreliable result. Effect and lowest amount of sensors can get parameters easily and lower the cost.

3. When the data are acquired from the same type of machines, the geometry accuracy is focused to make correctly adjustment, for building the machine. When heat errors occur, the accuracy is in the tolerance.

4. The core technology of the FANUC, HEIDENHAIN controller cannot be acquired that it can merely be compensated by external way. It is expected to get the core interpolation technology for the direct compensation in order to develop good software.

5. If the machine is 4 or 5 axes machine, the result of compensation will become very hard, due to adding tilting or rotating axis. In the future, it is necessary to do more study and experiment at multiple axes machine tools.

\section{Acknowledgements}

Thanks to Quaser machine tools supporting the hardware, software and others and the PMC's assistance and technology transfer to finish this paper.

\section{References}

1. R. Ramesh, M.A. Mannan, A.N. Poo, International Journal of Machine Tools \& Manufacture 40, 1235$1256(2000)$ 
2. R. Ramesh, M.A. Mannan, A.N. Poo, International Journal of Machine Tools \& Manufacture 40, 12571284 (2000)

3. J.H. Lee, Y. Liu, S.H. Yang, International Journal of Machine Tools \& Manufacture 46, 1508-1516 (2006)

4. J.S. Wu, Y.T. GONG, Machinery Monthly 25(3), 4858 (2003, In Chinese)

5. J.H. Lee, S.H. Yang, International Journal of Machine Tools \& Manufacture 42, 147-155 (2002)

6. P. Vanherck, J. Dehaes, M. Nuttin, Computers in industry 33, 119-125 (1997)

7. O. Horejša, M. Mareša, L. Novotnýa, Procedia CIRP 4, 67-72 (2012) 